\title{
Synthesis of Secosterols as an Arena for C-H Functionalization and C-C Manipulation Tactics
}

\section{Robert C. Heinze}

Philipp Heretsch*

Synlett 2017

The name of the corresponding author has been corrected. The editorial office apologizes for this mistake. 